\title{
The Galactic $511 \mathrm{keV}$ morphology and the old stellar population
}

\author{
M. Ali ${ }^{1 *}$, S.C. Ellis ${ }^{1,2}$, S. Sharma ${ }^{1}$, J. Bland-Hawthorn ${ }^{1}$ \\ ${ }^{1}$ Sydney Institute for Astronomy, \\ The University of Sydney NSW 2006, Australia. \\ E-mail: m.ali@physics.usyd.edu.au \\ ${ }^{2}$ Australian Astronomical Observatory, \\ P.O. Box 296, Epping NSW 1710, Australia.
}

\begin{abstract}
The source of Galactic positron annihilation emission is a mystery. What is known is that photons produced in positron-electron annihilation are highly concentrated at the Galactic centre, with a slightly off-centred peak with respect to Galactic longitude. Using synthetic models of the Milky Way we find possible connections between age-bound stellar structures in the Milky Way and the $511 \mathrm{keV}$ annihilation morphology. We find that in projection, the flux of the old tilted stellar bulge/bar can reproduce the Gaussian fit parameters and the apparent asymmetry observed in the radiation, supporting a large number of annihilation sources in an extended bulge/bar population. When looking at the same stars in the disk we were not able to reproduce the bulge to disk ratio in flux, indicating either a different class of sources in the disk or significant positron propagation out of the bulge.
\end{abstract}

The Extreme and Variable High Energy Sky - extremesky2011,

September 19-23, 2011

Chia Laguna (Cagliari), Italy

${ }^{*}$ Speaker. 


\section{Introduction}

The first report of $511 \mathrm{keV} \gamma$-rays coming from positron annihilation in astrophysical environments was from the Solar Maximum Mission (SMM) aboard the OSO-7 satellite [1]. Several years later a balloon-borne experiment discovered the same signal coming from within $15^{\circ} \mathrm{FWHM}$ of the Galactic centre (GC) [2]. Today, the INTEGRAL satellite observes the $511 \mathrm{keV}$ sky and, using the spectrometer SPI, it has the highest resolution $\left(\sim 3^{\circ}\right.$ FWHM [3]) of any instrument at these wavelengths measuring an annihilation rate of $2 \times 10^{43} \mathrm{e}^{+} \mathrm{s}^{-1}$ in the Milky Way. The distribution of the radiation is centred within $\sim 6^{\circ}$ of the galaxy with a bulge/disk positron annihilation rate of $\sim 1.4$ [4]. A recently discovered feature of the $511 \mathrm{keV}$ morphology is the asymmetry in radiation flux counts favouring negative Galactic longitudes over positive ones [5]. The exact nature and cause of this is largely unknown, its origin being suggested to arise in the disk [5], the bulge [6] or from flawed background treatment [7]. A disk asymmetry is suggested to dominate at $|l|>10^{\circ}$ and possible mechanisms include the Galactic distribution of the LMXB population [5] or spiral arm densities [8]. A bulge asymmetry is suggested to arise from a longitudinally off-GC peak in $511 \mathrm{keV}$, modelled at $l \approx-0.6^{\circ}, b \approx 0^{\circ}[6,9]$, although no possible physical mechanisms for this have been suggested.

While there have been attempts to unify candidate sources to annihilation observations [5], apart from the Sun, there have been no confirmed detections of discrete extraterrestrial sources of the radiation [10]. Theoretical stellar contributions to the Galactic positron budget are expected primarily from radioactive decay in supernovætype Ia (SN Ia) and pair production in the jets of low mass X-ray binaries (LMXBs) and microquasars ( $\mu \mathrm{Qs})$, with lesser contributions are expected from pulsars, millisecond pulsars (MSPs), supernovætype II (SN II), novæand high-mass X-ray binaries (HMXBs) [4]. Apart from SN II, these phenomena are connected by their evolutionary phases. For example, white dwarf progenitors in binary systems can accrete matter in such a way that they are observed as Novæor HMXBs and under the right conditions can suffer thermonuclear explosions as an SN Ia. Similarly neutron stars, conceived in SN II explosions, go on to produce more positrons in highly magnetised pulsar jets and in accreting binary systems, appearing as a MSPs, LMXB, HMXB or $\mu \mathrm{Qs}$. All of these positron-producing phenomena have lived Galactically long lives, some well in excess of 10 Gyrs, occupying the Galactic bulge/bar, thick disk and stellar halo. Without knowing which of these contribute the significantly to the Galactic positron budget, we have decided to approximate the density distribution of positrons produced by any class or classes of these stars by examining the number density distribution of stars in the aforementioned regions they occupy. This is the assumption that any combination of the sources will produce the same distribution, i.e. they are all old ( $\geq 10 \mathrm{Gyr}$ ) stars. Furthermore, many of these stellar phenomena exist in binaries and as Galactic binarity is a function of stellar density, we need only consider one to account for both.

Motivation for this analysis comes from the relatively poor resolution of experiments at these wavelengths compared to those in the optical and near infrared. Hipparcos and 2MASS are two such wide area surveys sampling large volumes of the Milky Way and providing high precision data of stars. Surveys such as these allow theoreticians to produce self consistent models of Galactic structure, including the tilted central bulge/bar [11], the long thin bar [12] or tufts on the bulge/bar [13], the thin disk (now with only two spiral major arms [14]), the thick disk and the stellar halo 
[15], all of which have interrelationships between position, age, metallicity, velocity dispersions etc. In order to exploit these models fully, we have used Galaxia [16]. A fast code for creating a synthetic survey of the Milky Way, it is able to generate a smooth distribution of stars over a given volume of the Galaxy allowing the specification of Galactic structure (e.g. the bulge/bar, thin disk, thick disk etc.) and other useful parameters (e.g. age, metallicity, velocity dispersion etc.).

Using Galaxia to probe population parameters, we can test under what conditions, if any, the $511 \mathrm{keV}$ emission arises from a statistically large number of sources in an extended population rather than a few discrete sources. This is performed by considering each star as a standard candle and using the distance to each star, we find a value for the flux per unit solid angle along a given line of sight, computing this for all lines of sight produces an all-sky map of relative flux density. This is repeated for various configurations of Galactic components and comparisons are made to the 511 $\mathrm{keV}$ morphology including the bulge/disk ratio and the longitudinal asymmetry $[5,6,9,17]$.

\section{Model}

In modelling the Milky Way, Galaxia, uses the same density functions as that of the Besançon model [15] and for generating stellar properties it uses Padova Isochrones [18] (see [16] for a detailed description of parameters). We have allowed the longitudinal tilt of the bulge/bar, $\alpha$, to vary through $11.1^{\circ}, 20^{\circ}$ and $30^{\circ}$, since the exact value is still a matter of discussion $[11,12,13,15]$.

A model of the distribution of stars in the Milky Way was generated for various parameters; each $\operatorname{star}(j)$ was divided by its heliocentric radius $(r)$ squared and totalled for all the stars $(N)$ in a given pixel $(i)$ in $l$ and $b$, this number represents the flux $\left[F_{i}(l, b)\right]$ of that pixel:

$$
F_{i}(l, b)=\sum_{j} \frac{1}{r_{j}^{2}}
$$

where the flux per unit solid angle is given by $F_{i}(l, b) / \Omega=F_{i}(l, b) /\left[\Delta l \int_{b_{\min }}^{b_{\max }} \sin (b) d b\right]$. This is a measure of the relative flux density observed at Earth for all the stars in the model.

In order to compare this to $511 \mathrm{keV}$ data, we use a similar fitting scheme outlined in Bouchet et al. (2010)[6]. Single and double 2D Gaussian fitting algorithms are employed for the bulge flux in $l$ and $b$ and another one is used for the disk. There are five fitting methods in the analysis:

1. Fit one Gaussian for the bulge: fix $l_{0}, b_{0}=0$, three free $\left(G_{1}, \sigma_{1 l}, \sigma_{1, b}\right)$.

2. Fit two Gaussians for the bulge: fix $l_{0}, b_{0}=0$, six free $\left(G_{1}, \sigma_{1 l}, \sigma_{1 b}, G_{2}, \sigma_{2 l}, \sigma_{2 b}\right)$.

3. Fit two Gaussians for the bulge using the $\sigma$ values from 2 , four free $\left(G_{1}, l_{0 B}, G_{2}, b_{0 B}\right)$.

4. Fit one Gaussian for the disk, five free $G_{3}, \sigma_{3 l}, \sigma_{3, b}, l_{0 D}, b_{0 D}$.

5. Normailise: fit three Gaussians for the bulge+disk using 3 and 4 , three free $G_{1}, G_{2}, G_{3}$.

For the fitting routine, a non-linear least squares algorithm was employed. Equation 2.2 is the most general form of the Gaussians used for the fit (including the disk),

$$
f(l, b)=G_{1} e^{-\left(\frac{\left(l-l_{0 B}\right)^{2}}{2 \sigma_{1 l}^{2}}+\frac{\left(b-b_{0 B}\right)^{2}}{2 \sigma_{1 b}^{2}}\right)}+G_{2} e^{-\left(\frac{\left(l-l_{0 B}\right)^{2}}{2 \sigma_{2 l}^{2}}+\frac{\left(b-b_{0 B}\right)^{2}}{2 \sigma_{2 b}^{2}}\right)}+G_{3} e^{-\left(\frac{\left(l-l_{0 D}\right)^{2}}{2 \sigma_{3 l}^{2}}+\frac{\left(b-b_{0 D}\right)^{2}}{2 \sigma_{3 b}^{2}}\right)},
$$



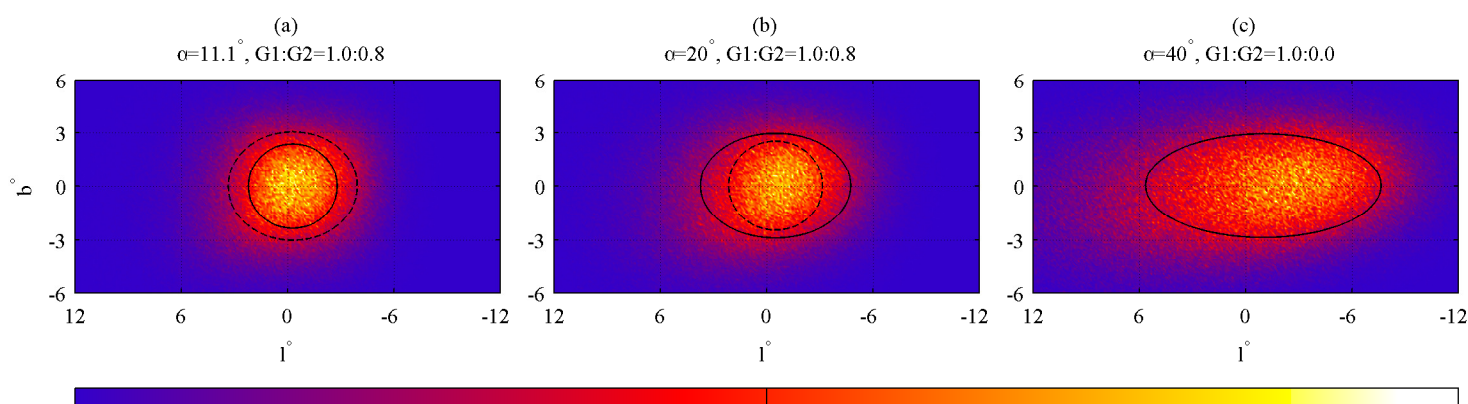

0.5

Figure 1: Galaxia data showing the relative flux per unit solid angle of the bulge, normalised between 0 and 1 , in $0.1^{\circ}$ bins. The lines indicate the FWHMs of the fitting function in Method 3, solid represents the greater amplitude Gaussian.

where $G_{1}$ and $G_{2}$ define the amplitude of the bulge and $G_{3}$ defines the amplitude of the disk. Apart from the amplitude constants, which only have relative significance, these parameters can be compared to their established counterparts in the annihilation morphology including the bulge/disk ratio and the longitudinal asymmetry $[5,6,9,17]$. Which were found by calculating the total flux in the corresponding regions along $l$ and $b$ via the the surface integral:

$$
F_{\text {tot }}(l, b)=\int_{b_{\min }}^{b_{\max }} \int_{l_{\min }}^{l_{\max }} \sin (b) f(l, b) d l d b .
$$

The bulge to disk ratio is found through Equation (2.3) for the bulge Gaussians $G_{1}$ and $G_{2}$ separately from the disk Gaussian $G_{3}$ and the asymmetries are computed in the regions $|b|<10^{\circ}$ for $-50^{\circ}<l<0^{\circ}$ vs. $0^{\circ}<l<50^{\circ}$ and $-180^{\circ}<l<-50^{\circ}$ vs. $50^{\circ}<l<180^{\circ}$ (as in [5, 6]).

\section{Results}

We begin in Galaxia by selecting all bulge/bar sources and examine their stellar morphology. As previously mentioned, each star is considered to be a standard candle and the relative flux in $l, b$ is calculated. Figure 1 shows a contour map of the data superimposed with the best fit Gaussians from Method 3 for various tilt angles of the bulge/bar. The Gaussian centres are shifted to negative longitudes in all chosen tilt angles, the FWHMs are confined within $15^{\circ}$ of the centre and we produce greater flux in negative longitudes compared to positive ones. All these values, summarised in Table (1), are either compatible with or at least similar to those obtained for the 511 $\mathrm{keV}$ radiation. As we have used a semi-continuous stellar density model, these results support a large number of annihilation sources in an extended bulge/bar population.

In fitting the disk via Method 4, we explore three age regions in Galaxia. 1) a young disk (0-7 Gyrs), 2) an old disk (10-14 Gyrs) and 3) the entire Milky Way (0-14 Gyrs). Note that an old disk corresponds to the same class of stars as in the bulge/bar. The best-fit parameters of the disk are shown in Table 1, although it is difficult to make comparisons to the $511 \mathrm{keV}$ morphology when fitting these parameters due to the low surface brightness of the disk. Although, this is not unexpected as the underlying disk geometry of the radiation is not well understood, for example there

\begin{tabular}{|c|c|c|c|c|c|c|c|c|}
\hline \multirow{2}{*}{ Reference } & \multicolumn{5}{|c|}{ bulge model } & \multicolumn{3}{|c|}{ disk model } \\
\hline & $\alpha$ tilt $^{\circ}$ & $G_{1}: G_{2}$ & $l_{0 B}, b_{0 B}$ & $\mathrm{FWHM}_{1 l, 1 b, 2 l, 2 b}^{\circ}$ & $F_{l-}^{50} / F_{l+}^{50^{6}}$ & Age (Gyrs) & $l_{0 D}, b_{0 D}$ & $\mathrm{FWHM}_{3 l, 3 k}^{\circ}$ \\
\hline Method $3+4$ & 20.0 & $1.00: 0.76$ & $-0.51,0.02$ & $8.5,5.9,5.3,5.0$ & 1.3 & $10-14$ & $-0.21,-0.06$ & $31.8,7.7$ \\
\hline Knödlseder (2005) [9] & & & $-0.6_{-0.3}^{+0.3}, 0.1_{-0.3}^{+0.3}$ & $7.2_{-0.9}^{+0.9}, 8.1_{-0.9}^{+0.9}$ & & & & \\
\hline Bouchet (2010) [6] & & & $-0.64_{-0.19}^{+0.20}, 0.06_{-0.20}^{+0.19}$ & $3.2_{-1.0}^{+1.0}, 11.8_{-1.5}^{+1.9}$ & $1.1_{-0.4}^{+0.4}$ & & & \\
\hline Weidenspointner (2008) [5] & & & & & $1.8_{-0.3}^{+0.5}$ & & & \\
\hline
\end{tabular}

Table 1: Model fit parameters for: 1) The Bulge/Bar, shown in Figure 1, computed within $|l|,|b|<30^{\circ}$, flux ratios are listed for $|b|<10^{\circ}$. 2) The Disk, shown in Figure 2. 


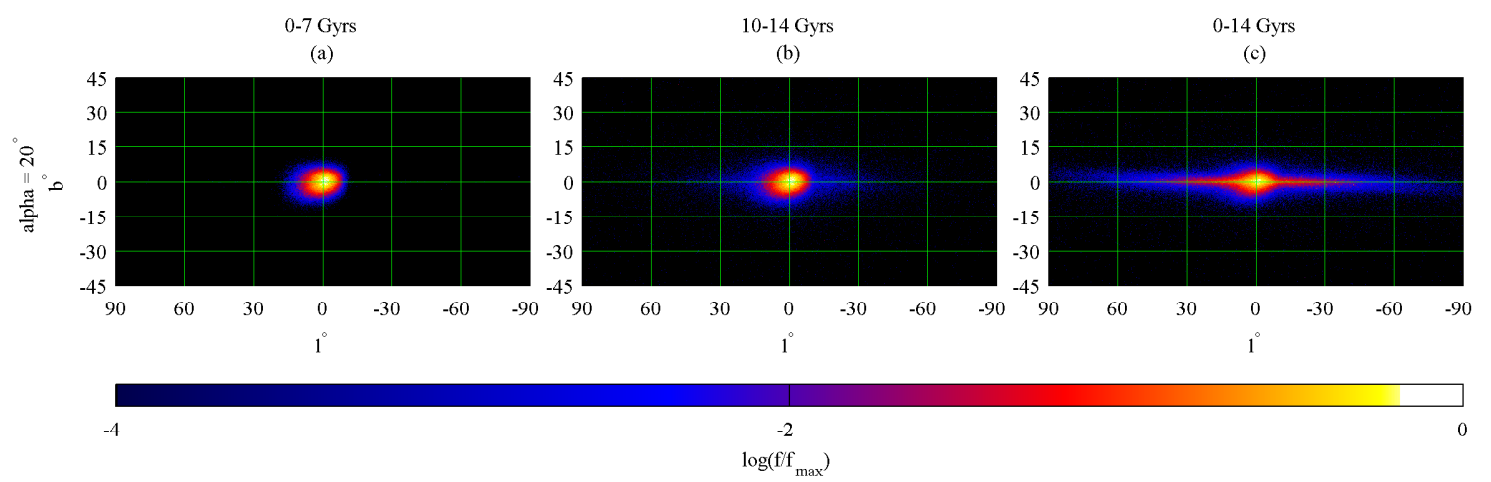

Figure 2: Galaxia data for various age regions showing the logarithm of the relative flux per unit solid angle in $0.1^{\circ}$ bins, the tilt angle of the bulge/bar is $\alpha=20^{\circ}$. The peak flux is normalised to zero and the data is restricted to the interval $-4<\log \left(f / f_{\max }\right)<0$ as in [6].

have been suggestions that support either a thick disk component and a small bulge or alternatively a thin disk component and an extended bulge [4]. In any case, we have compared our results to published $511 \mathrm{keV}$ analogues for the bulge to disk ratio of annihilation flux as well as the possible inner and outer longitudinal disk flux asymmetry.

Figure 2 and Table 2 summarise the results from Method 5, which is a bulge + disk composite analysis. They indicate that the stellar densities of both young or old disks are insufficient in producing the required disk-flux of the radiation alone, it is only when including all of the stars in the Milky Way disk that it can. This shows that, in the case of nearby annihilation, the old source/s responsible for the bulge flux cannot account for the disk flux as well. There must be at least one other distinct class of sources responsible for the rest of the annihilation flux. In the case of significant positron propagation this could be explained by positrons escaping the bulge/bar into the disk and annihilating there, although this seems unlikely [8].

This investigation shows that the flux produced by the old stellar distribution of the Galactic bulge/bar in projection can provide a natural explanation of the bulge morphology observed in 511 $\mathrm{keV}$ data, supporting a large number of annihilation sources in an extended bulge/bar population. It is still unclear whether we should expect to see positrons being produced by many sources in the bulge/bar and annihilating nearby or if we should see few sources of positrons that annihilate uniformly within the bulge/bar volume. In terms of a many positron source case, this is not surprising as many of the stars thought to produce significant quantities of positrons are old and hence occupy the stellar bulge/bar. For example, pulsars accreting in binary systems can produce millisecond pulsars, LMXBs, HMXBs and microquasars, all of which are considered to produce significant quantities of positrons. Furthermore, as binarity increases with stellar density, the bulge/bar is likely to contain many of these classes of stars. A distribution in the disk of the same old sources does not provide the flux required for the observed bulge to disk ratio in $511 \mathrm{keV}$, suggesting that

\begin{tabular}{|c|c|c|c|c|c|c|c|c|c|}
\hline \multirow{2}{*}{ Reference } & \multicolumn{5}{|c|}{ bulge + disk } & \multicolumn{4}{|c|}{ asymmetry $\left(|b|<10^{\circ}\right)$} \\
\hline & Age (Gyrs) & $G_{1}: G_{2}: G_{3}$ & {$\left[F_{B 1}, F_{B 2}\right], F_{D}$} & $F_{\text {tot }}$ & $\mathrm{B} / \mathrm{D}$ & $F_{-}^{50^{\circ}}$ & $F_{+}^{50^{\circ}}$ & $F_{-}^{50^{\circ}} / F_{+}^{50^{\circ}}$ & $F_{-}^{180^{\circ}} / F_{+}^{180^{\circ}}$ \\
\hline \multirow{2}{*}{$\begin{array}{c}\text { Method 5 } \\
\alpha=20^{\circ}\end{array}$} & $10-14$ & $1.00: 0.63: 0.02$ & $13.4,4.4,2.2$ & 20 & 8.0 & 4.3 & 3.4 & 1.3 & 1.0 \\
\hline & $0-14$ & $1.00: 0.37: 0.37$ & $9.6,1.8,8.6$ & 20 & 1.3 & 4.1 & 3.6 & 1.2 & 1.0 \\
\hline \multicolumn{3}{|c|}{ Knödlseder (2005) [9] } & {$\left[10.5_{-0.6}^{+0.6}\right], 7_{-4}^{+4}$} & $15-29$ & $0.2-3.3$ & & & & \\
\hline \multicolumn{3}{|c|}{ Prantzos (2011) [4] (bulge + thick disk) } & $4.8_{-0.4}^{+0.7}, 2.7_{-0.4}^{+0.9}, 9.4_{-1.4}^{+1.8}$ & 17.1 & 0.8 & & & & \\
\hline \multicolumn{3}{|c|}{ Prantzos(2011) [4] (bulge + thin disk) } & {$\left[21.4_{-1.2}^{+1.1}\right], 7.3_{-1.9}^{+2.6}$} & 28.7 & 2.9 & & & & \\
\hline
\end{tabular}

Table 2: Best-fit parameters and comparisons to the bulge + disk model shown in Figure 2 along with the bulge/disk ratios and longitudinal asymmetry. The fluxes are listed in units $10^{-4} \mathrm{ph} \mathrm{cm}^{-2} \mathrm{~s}^{-1}$, the total flux has been normalised to $20 \times 10^{-4} \mathrm{ph} \mathrm{cm}^{-2} \mathrm{~s}^{-1}$ and the interval $|F|<50^{\circ}$ is normalised to $7.7 \times 10^{-4} \mathrm{ph} \mathrm{cm}^{-2} \mathrm{~s}^{-1}$ from [6]. 
there may a different class of sources in the disk. However, it is also possible that there is significant propagation of bulge positrons into the disk instead.

In conclusion, we have reproduced the $511 \mathrm{keV}$ morphology, including Gaussian fit parameters, the bulge/disk ratio and the apparent asymmetry, based on models of the Galactic stellar density and in doing so find a possible link to the old spheroid population.

\section{References}

[1] Chupp, E.L. et al. Solar Gamma Ray Lines observed during the Solar Activity of August 2 to August 11, 1972. Nature, 241 (1973) 333.

[2] Leventhal, M. et al. Detection of 511 kev positron annihilation radiation from the galactic center direction. APJ, 225 (1978) L11.

[3] Weidenspointner, G. et al. Positron astronomy with spi/integral. NAR, 52 (2008) 454.

[4] Prantzos, N. et al. The 511 kev emission from positron annihilation in the galaxy. RMP, 83 (2011) 1001.

[5] Weidenspointner, G. et al. An asymmetric distribution of positrons in the galactic disk revealed by $\gamma$-rays. Nature, 451 (2008) 159.

[6] Bouchet, L. et al. On the morphology of the electron-positron annihilation emission as seen by spi/integral. APJ, 720 (2010) 1772.

[7] Churazov, E. et al. Positron annihilation spectrum from the galactic centre region observed by spi/integral. MNRAS, 357 (2005) 1377.

[8] Higdon, J.C. et al. The galactic positron annihilation radiation and the propagation of positrons in the interstellar medium. APJ, 698 (2009) 350.

[9] Knödlseder, J. et al. The all-sky distribution of 511 keV electron-positron annihilation emission. $A \& A, 441$ (2005) 513.

[10] de Cesare, G. Searching for the $511 \mathrm{keV}$ annihilation line from galactic compact objects with the IBIS gamma ray telescope. A\&A, 531 (2011) A56.

[11] López-Corredoira, M. et al. A boxy bulge in the milky way. inversion of the stellar statistics equation with 2 mass data. A\&A, $\mathbf{4 3 9}$ (2005) 107.

[12] López-Corredoira, M. et al. The long bar in the milky way: Corroboration of an old hypothesis. The Astronomical Journal, 133 (2007) 154.

[13] Martinez-Valpuesta, I. et al. Unifying A Boxy Bulge and Planar Long Bar in the Milky Way. APJL, 734 (2011) L20.

[14] Churchwell, E. et al. The spitzer/glimpse surveys: A new view of the milky way. PASP, 121 (2009) 213.

[15] Robin, A.C. et al. A synthetic view on structure and evolution of the Milky Way. A\&A, 409 (2003) 523.

[16] Sharma, S. et al. Galaxia: A Code to Generate a Synthetic Survey of the Milky Way. APJ, 730 (2011) 3.

[17] Churazov, E. et al. Positron annihilation spectrum from the galactic centre region observed by spi/integral revisited: annihilation in a cooling ism? MNRAS, 411 (2011) 1727.

[18] P. Marigo et al. Evolution of asymptotic giant branch stars. A\&A, 4823 (2008) 883. 